Thesis

\title{
Is the Portable Chest Radiographic More Reliable to Reveal Covid19 in Highly Suspicion Patient before Real-Time Reverse Transcription Polymerase Chain Reaction (RT-PCR) Test?
}

\author{
Bushra A. A. Albazi ${ }^{* 1}$, Dr Noof. Albaz ${ }^{2}$, Dr Nayef.Alqahtani1 ${ }^{3}$, Dr. Angham Salih ${ }^{4}$, Dr Rafat Mohtasab ${ }^{5}$ \\ ${ }^{1}$ Radiology Imaging Diagnostic, PSMMC, RIYADH 11159/7879, Kingdom of Saudi Arabia, Senior Radiograher \\ ${ }^{2}$ College of Medicine, King Saud bin Abdulaziz University for Health Sciences, Riyadh 11481/3660, \\ Kingdom of Saudi Arabia, King Abdullah International Medical Research Centre, Riyadh, Saudi Arabia \\ ${ }^{3}$ Radiology Imaging Diagnostic, Prince Sultan Medical Military City, Riyadh Consultant and Head of Chest \\ Imaging Division \\ ${ }^{4}$ Radiology Imaging Diagnostic, Prince Sultan Medical Military City, Riyadh Radiologist Senior Registrar \\ ${ }^{5}$ Medical Imaging Consultant SCFHS, Al-Faisal University, RIYADH 11533/50927 Kingdom of Saudi Arabia \\ *Corresponding author: Bushra A. Albazi; balbazi@psmmc.med.sa
}

Received 14 February 2021;

Accepted 02 March 2021;

Published 17 March 2021

\begin{abstract}
A large number of patients with coronavirus disease 2019 (COVID-19) present at hospitals. There are a limited number of isolation rooms open, and patients must often wait a long time to get a reverse transcription-polymerase chain reaction (RT-PCR) test done. This necessitates the introduction of effective triage plans. A patient with suspicions is referred to an emergency room (ED) depending on their medical record for a simple physical assessment, blood test findings, and chest imaging.A retrospective study design was conduct at Prince Sultan Medical Military City (PSMMC). Ethical approval was obtained from the institutional board to wave the consent forms since it is a retrospective study. Only the primary investigator has had the data access to the patients' medical records. The collected patient records were under specific categories, including symptoms score starts from 5 and above, RT-PCR test result done after CXRP imaging, the patient admitted to the emergency department (ED). Excluding all CXRP done after RT-PCR TEST, positive Covid 19 admitted to the intensive care unit (ICU), pediatric patients, and patients with score symptoms were less than five. Two experienced radiologists reviewed the images blindly, and the inter-observer reliability of observations noted by the radiologists was calculated.

As for the relationship between the x-ray reading and the RT-PCR test result, our results showed a high correlation between the variables (chi-square $\chi^{2}=12.44$, with $\mathrm{df}=1$, and $\mathrm{p}<0.001$ ). The sensitivity of $\mathrm{x}$-ray diagnosing covid19 was $65.52 \%$, while the specificity was $54.51 \%$, and the accuracy of radiologists reading was $58.17 \%$. Furthermore, the positive predictive value (PPV) was $41.76 \%$, and the negative predictive value (NPV) was $76.05 \%$. Finally, the false positive rate (type-i error (alpha) was $45.49 \%$, and the false-negative rate (type-ii error (beta) was $34.48 \%$

Our research findings show that CXRP imaging can detect COVID-19 infection in symptomatic patients and can be a valuable addition to RT-PCR testing. In an inpatient ED environment where availability of test kits, laboratory equipment, and laboratory personnel is compromised and risks delaying patient treatment and hospital workflow, serial CXRP could theoretically be used as an adjunct diagnostic function and monitoring in patients suspected of having COVID-19.
\end{abstract}

Keyword: chest portable, chest portable, real-time reverse transcription polymerase chain reaction, emergency department

\section{Introduction}

A vast number of patients with a suspicion of coronavirus disease 2019 (COVID-19) arrive at hospitals, in which limited isolation rooms are available, and sometimes wait a long time to have a reverse transcription-polymerase chain reaction (RT-PCR) test performed. This calls for the implementation of efficient triage plans. A suspicions covid19 patient is admitted to an emergency department (ED) based on their medical record for, a simple 
physical examination, blood test results, and chest imaging will be performed.

Many reports have shown that chest computed tomography (CT) has a high sensitivity in the screening and diagnosis of COVID-19 pneumonia (Carotti et al., 2020; Ye, Zhang, Wang, Huang, \& Song, 2020; Yin et al., 2020). However, CT could be oversensitive, as it does not detect features unique of COVD19, especially during its initial stage (Akçay, Özlü, \& Yılmaz, 2020). The order of CT scans required personal protective equipment (PPE) by the radiology department staff, and the risk of transmitting the virus by clustering infected and noninfected patients is high. Moreover, a long waiting time interval exists during the post examination to sterilize the room and equipment.

Now a day, the American College of Radiology (ACR) suggests that the modality of using portable chest radiograph as a frontline tool approach for both confirm and suspicious patients could be considered to minimize the risk of cross-infection. Furthermore, CT should be used in hospitalized patients, based on specific clinical indication (RADIOLOGY, 2020).

The British Society of Thoracic Imaging (BSTI) has also recommended that a combination of clinical, laboratory and portable chest X-ray (CXRP) findings could be used to triage patients with a suspicion of COVID-19 in line with hospital admission recommendations, and CT should be reserved for more challenging scenarios (Khan et al., 2020). In specific clinical situations, the Ministry of Saudi health $(\mathrm{MOH})$ advised that portable chest radiography is recommended for easy cleaning portable machine and slight risk for contamination (HEALTH, 2020).

The best way to set an imaging basis for patients who are admitted to the hospital, taking into account the limitation of the RT- PCR test laboratories, meanwhile CXRP would be safe and functional under particular clinical circumstances (Bharadwaz \& Langfeldt, 2020). When RT-PCR testing is inaccessible and/or during the window time when RT-PCR test results are awaited, CXRP could be the only usable tool. It is also related that the normal CXR characteristics are known to both radiologists and other physicians and understood in the sense of suspect or confirmed COVID19 status (Bharadwaz \& Langfeldt, 2020).

Based on the aforementioned information, we think that research will help categorize the use of portable $\mathrm{x}$-ray and investigate CXRP reliability to diagnose COVID19 at ED. Therefore, this study aims to know if the portable chest radiographic is reliable to reveal COVID19 on a highly suspicious patient before the reverse transcription-polymerase chain reaction (RT-PCR) test. Generally, the rationale of this study is to investigate if the $\mathrm{X}$-ray equipment and facilities are available in the basic health units. Besides, this study will enhance our clinicians understanding of CXR findings in suspected COVID19 patients.

\subsection{Study objectives}

To investigate the reliability of CXRP in diagnosing COVID19 before RT-PCR test. Also to investigate the correlation between CXRP and RT-PCRT test.

\section{Literature review}

In December 2019, an epidemic started and is still ongoing in Wuhan, China. Since then, this unbelievably contagious COVID19 has been spreading internationally, with the number of deaths growing exponentially. The World Health Organization (WHO) publicly declared COVID19 as a pandemic on March 11th, 2020. The new COVID19-infected pneumonia is measured as fever, fatigue, dry cough and dyspnea ((Kooraki, Hosseiny, Myers, \& Gholamrezanezhad, 2020).

The majority of patients with COVID19 pneumonia have fever as the first symptom, and most of them have common chest imaging manifestations of viral pneumonia (Liu et al., 2020). Therefore, imaging methods are at the forefront of diagnostic decision-making, tracking progress, and evaluating potential complications (Lovas et al., 2020).

CXRP and CT scans are the standard imaging diagnostic measures for pneumonia (Pereira, Bertolini, Teixeira, Silla, \& Costa, 2020).CXR has not however been validated for its prognostic usefulness in analyzing coronavirus disease patients COVID19, and CXR is not considered susceptible to lung involvement detection at the early stage of the disease (Toussie et al., 2020). It is proven that the CXRP can be a valuable diagnostic tool in the current emergency setting for monitoring the rapid progression of lung abnormalities in infected patients, particularly in intensive care units (Borghesi \& Maroldi, 2020).However, in demonstrating these improvements, CT scans are more sensitive than a chest X-ray (Fatima, Ratnani, Husain, \& Surani, 2020). Bilateral, Multifocal, Multilobar ground glass, opacification with patchy convergence, and peripheral/subpleural or posterior distribution (or both) primarily in the lower lobes are the usual radiological features of COVID19 (Hu \& Wang, 2020). The most widely used tool for detecting and tracking lung irregularities is potentially CXRP (Jacobi, Chung, Bernheim, \& Eber, 2020).CXRP may play a role in diagnosing patients with suspected coronavirus 2 (SARS-CoV-2) infection with a serious acute respiratory syndrome, but only a few small-scale studies are available (Schiaffino et al., 2020). SARS-CoV-2 positive patients were the subject of most COVID19 studies (Singer et al., 2020). In 75\% of patients with reported SARS-CoV-2 infection, chest radiography revealed lung anomalies, ranging from $63.3 \%$ to $83.9 \%$, respectively, at 0-2 days and >9 days from the onset of symptoms (Vancheri et al., 2020). In critically ill patients with COVID19 caused pneumonia, CXRP is useful for screening possible thoracic symptoms, thereby initiating accelerated management. Therefore, it is very informative for the resuscitation personnel, and the surgeon as the preliminary test is conducted in intensive care units and the immediate postoperative course. The COVID19 crisis shows that a required method for patient care is portable radiography. With the promotion of new digital technologies and improved preparation and expanded knowledge of radiology technicians, CXRP can now restore confidence after a time of neglect (de Barry, Obadia, El Hajjam, \& Carlier, 2020).

In summary, Few studies have been carried out addressing the same issue(Bharadwaz \& Langfeldt, 2020; Borghesi \& Maroldi, 2020; Cozzi et al., 2020; Durrani, Haq, Kalsoom, \& Yousaf, 2020); this study will approach the subject differently to enrich the literature and provide valuable data. This study can fill in the gap in the literature regarding the evaluation of CXR portable image reliability to reveal COVID19.

\section{Materials and Methods}

\subsection{Study Design and participant}

A retrospective study design was conduct at Prince Sultan Medical Military City (PSMMC). Ethical approval obtained from the institutional board to wave the consent forms since it is a retrospective study. Only the primary investigator has had the data access to the patients' medical records. Three hundred forty-nine patient's records were collected from the hospital medical record department. The collected patient records were under specific 
categories, including symptoms score starts from 5 and above, RTPCR test result done after CXRP imaging, the patient admitted to the emergency department (ED). Excluding all CXRP done after RT-PCR TEST, positive Covid 19 admitted to the intensive care unit (ICU), pediatric patients, and patients with score symptoms were less than five. Two experienced radiologists reviewed the images blindly, and the inter-observer reliability of observations noted by the radiologists was calculated. CXRP imaging was extracted from a picture archiving and communication system (PACS). Two experienced radiologists participated in rereading the image and giving an initial report based on a negative Covid 19 , i.e., the patient's images were clear and positive Covid 19. So, all patients in this study have symptoms notice on the images based on radiologist scoring reading. The numbers of patient's images were divided between each radiologist. The only information shared with them was the patient's score number when s/he admitted to ED.

\subsection{Portable X-Ray}

In this study, the radiographers used Portable X-ray machines to screen the patients in ED triage. These portable X-ray machines come in a stand with wheels, while others are motorized. The stand with wheels allows technologists to move the machines around easily. Moreover, the machines do not require an electrical plug-in to be completely functional. Many portable X-ray machines facilitate taking patient's images by the technologists in different positions like stand, setting, and laydown. Also, the portable X-ray machines are known for reducing image processing speed and minimizing lengthy waiting periods, i.e., it takes less than 20 minutes for portable $\mathrm{X}$-ray machines to process an image (Yanagawa, Ohsaka, Oode, \& Omori, 2019). Having short processing time allows the radiologist to produce patients reports, making it easy to access by other health care providers in real-time. Besides, having a workstation attached to the portable X-ray machines, the technologists can boost the digital medical imaging that has been captured out of the field. However, digital imaging technologies' efficiency has helped reduce X-ray test retakes and provide patient-related information that is fast and precise. There is no need for patients and technologists to wait for the unit to produce the films and worry about bad image quality. In other words, radiologists and patients will get reliable and quick outcomes with portable X-ray machines without risking patients coming to Radiology emergency departments to do the $\mathrm{X}$-ray images.

\subsection{Radiologist scoring system to read the image.}

To give each patient in this study a scoring number, the radiologists have to demonstrate two steps. These two steps in imaging processing are used in the CXR scoring system. The first step is to sepreate each lung into three frontal chest projection zones (PA or $\mathrm{AP})$, labelled with the right lung letters $\mathrm{A}, \mathrm{B}$, and $\mathrm{C}$, while the left lung letters D, E, and F (See figure 1).

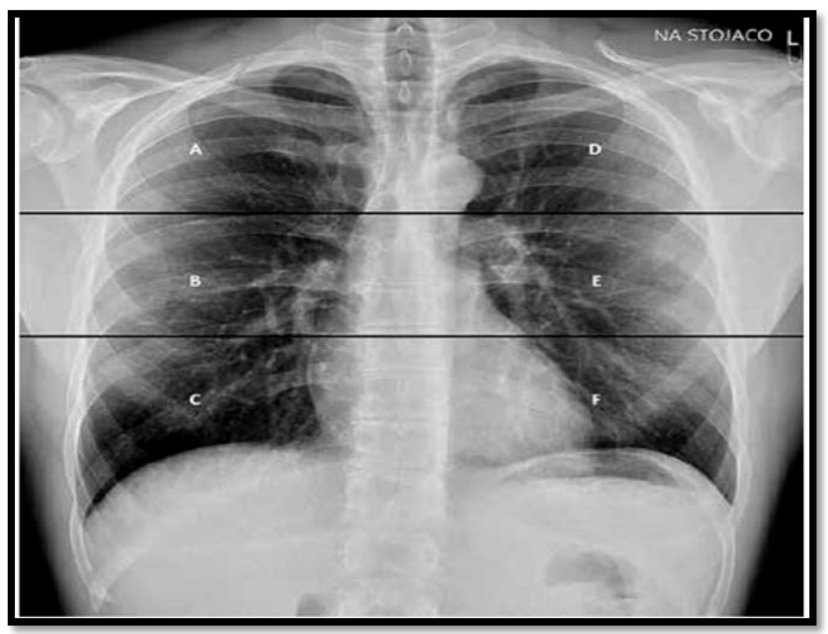

Figure 1: Chest Project Zone

The letters separate lungs into three levels: upper level, A, and D (above the lower wall of the aortic arch). The middle level includes $\mathrm{B}$ and $\mathrm{E}$ (under the lower wall of the aortic arch. Finally, above the lower wall of the more inferior right lung vein (i.e., the hilar structures).

The second step consists of assigning the labels (from 0 to 3 points) to each zone based on the observed lung abnormalities.It includes the following codes: 0 -no lung abnormalities,1-interstitial infiltrations, 2 -interstitial and alveolar infiltrate (See figure 2 and 3).

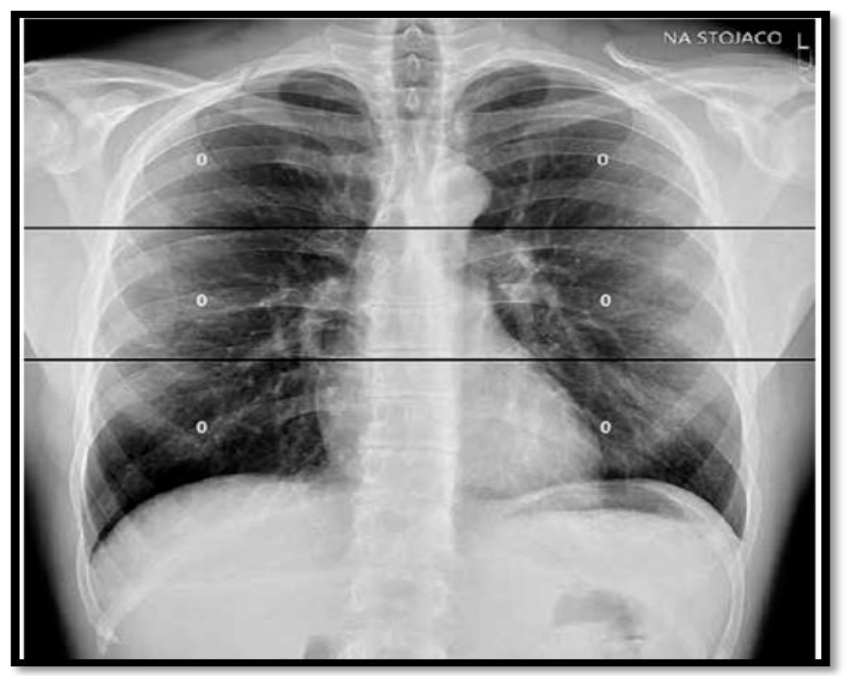

Figure 2: Observe Lung Abnormality Zone

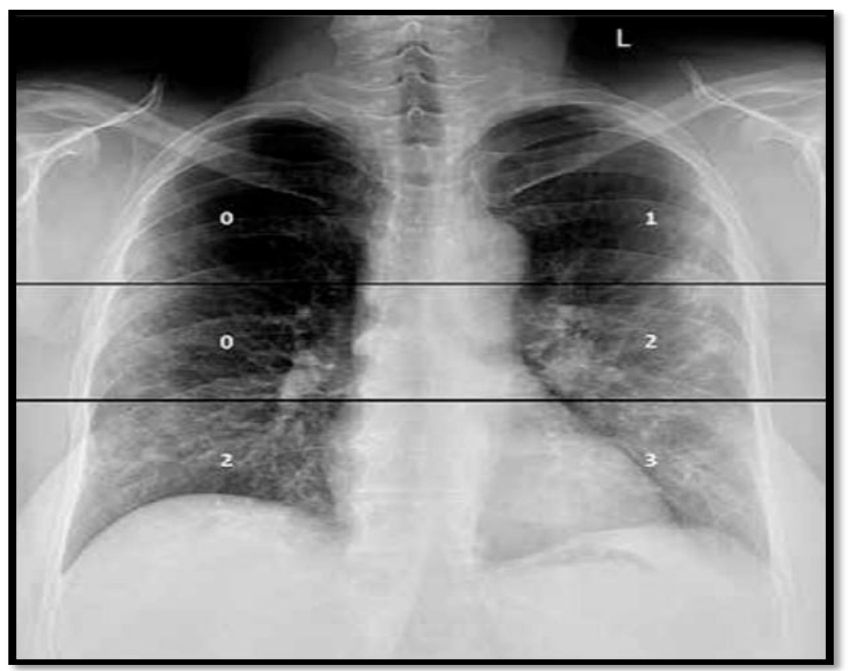

Figure 3: Complete Reporting Zone Abnormility 
It is worth highlight that the above two steps were adopted from Borghesi and Maroldi (2020). According to them, it will be easy to see the complete CXR and partial score for each region (Borghesi \& Maroldi, 2020).

\subsection{Statistical analysis}

Descriptive statistics were used in this study to explore the relationship between imaging and these factors after radiologists reporting all images. Chi-square analysis was used to investigate the correlation between the variable (RT-PCR TEST - Images reporting), and the level of significance set at a P-value of 0.05 .

\section{Results and Discussion}

All patients had CXRP at first admit to ED, and later within 24 hours, they had RT-PCR test for COVID19. The result showed a
$12 \%$ different result between radiologist reading images and RTPCR test result. Radiologist reading was not COVID19, and the test result was positive. In the other hand, different result 30\% show radiologist reading for a patient as COVID19 and RT-PCR test result is negative. When look deep in the data collection after calculate all the variable and analysis. In the first different percentage, $12 \%$ of patients score symptoms were from five until seven, and none of the patients had fever symptoms or dry cough and medical history of lung disease. On other $30 \%$ differently most of the patient having high score symptoms from 8 and above with medical history of lung disease along with fever, headache, running nose, dihorrah, sore throught, active chest pain, shortness of breathing and vomiting see figure (4).

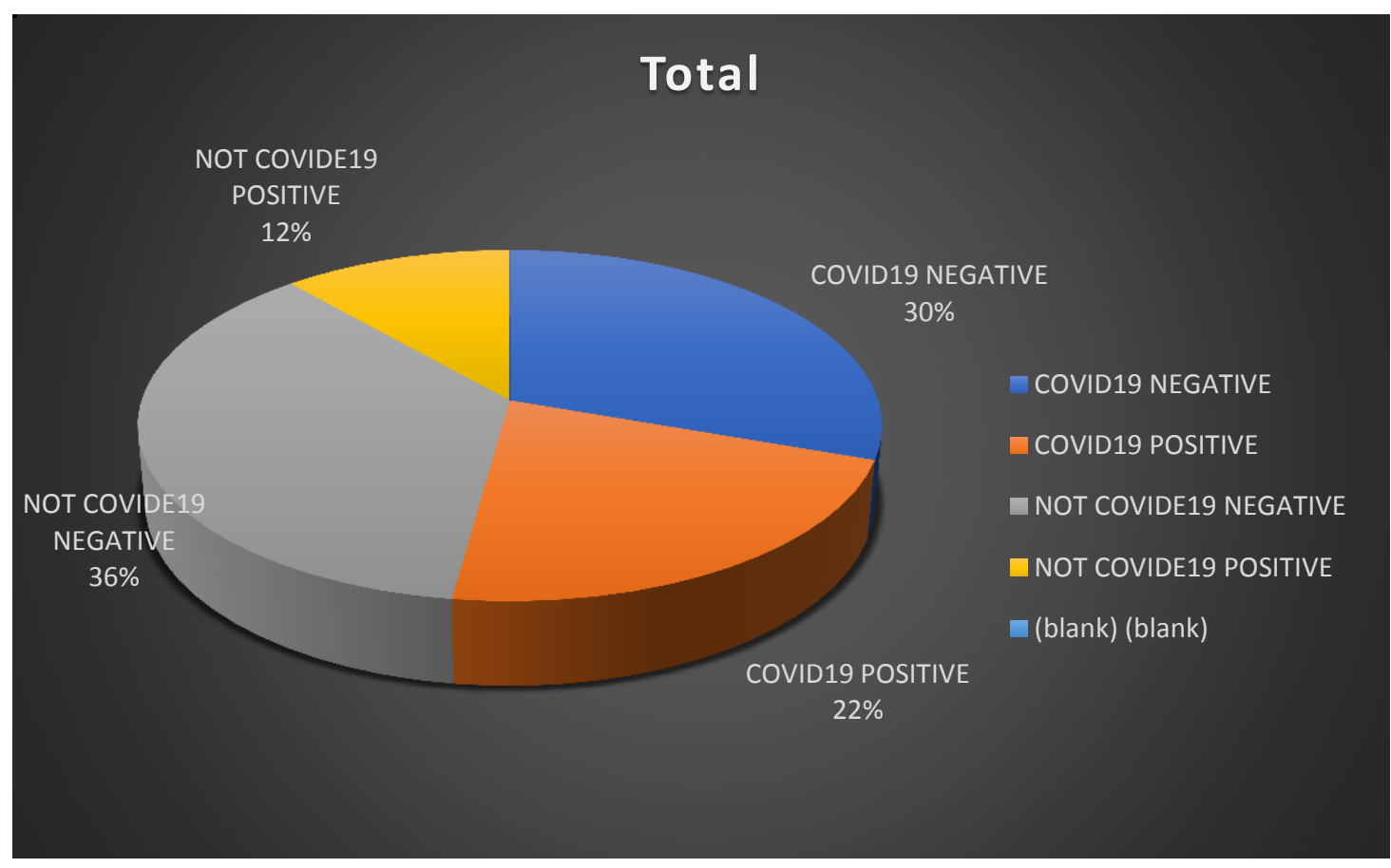

Figure 4: Radiologist Reading and RT-PCR Test

Table 1: Radiographic Feature and Distribution

\begin{tabular}{|c|c|c|c|c|c|c|c|c|}
\hline \multirow[b]{3}{*}{ radiographic feature } & \multirow{3}{*}{$\begin{array}{l}\text { Level } \\
\text { Abnormal CXR }\end{array}$} & \multirow{3}{*}{\begin{tabular}{l|} 
Count \\
46
\end{tabular}} & \multirow{3}{*}{\begin{tabular}{|r|} 
Total \\
349
\end{tabular}} & \multirow{2}{*}{\multicolumn{2}{|c|}{ Proportion }} & \multirow{3}{*}{$\begin{array}{l}\mathbf{p} \\
<.001\end{array}$} & \multicolumn{2}{|c|}{ 95\% Confidence Interval } \\
\hline & & & & & & & \multirow{2}{*}{$\begin{array}{l}\text { Lower } \\
0.09814\end{array}$} & \multirow{2}{*}{$\frac{\text { Upper }}{0.1719}$} \\
\hline & & & & 0.132 & $13 \%$ & & & \\
\hline & Reticular-nodular opacities & 30 & 349 & 0.086 & $9 \%$ & $<.001$ & 0.05875 & 0.1204 \\
\hline & Grounded glass opacities & 33 & 349 & 0.095 & $10 \%$ & $<.001$ & 0.06598 & 0.1302 \\
\hline & consolidation & 44 & 349 & 0.126 & $13 \%$ & $<.001$ & 0.09312 & 0.1655 \\
\hline & pneumothorax & 29 & 349 & 0.083 & $8 \%$ & $<.001$ & 0.05635 & 0.1172 \\
\hline & Normal & 167 & 349 & 0.479 & $48 \%$ & 0.454 & 0.42505 & 0.5323 \\
\hline \multirow[t]{9}{*}{ distrubition } & Peripheral & 36 & 349 & 0.103 & $10 \%$ & $<.001$ & 0.07330 & 0.1399 \\
\hline & Perihilar & 7 & 349 & 0.020 & $2 \%$ & $<.001$ & 0.00810 & 0.0409 \\
\hline & Diffuse & 42 & 349 & 0.120 & $12 \%$ & $<.001$ & 0.08813 & 0.1592 \\
\hline & Basal predominance & 22 & 349 & 0.063 & $6 \%$ & $<.001$ & 0.03992 & 0.0939 \\
\hline & Superior predominance & 25 & 349 & 0.072 & $7 \%$ & $<.001$ & 0.04689 & 0.1039 \\
\hline & Right lung & 17 & 349 & 0.049 & $5 \%$ & $<.001$ & 0.02863 & 0.0768 \\
\hline & Left lung & 24 & 349 & 0.069 & $7 \%$ & $<.001$ & 0.04455 & 0.1006 \\
\hline & Bilateral & 9 & 349 & 0.026 & $3 \%$ & $<.001$ & 0.01186 & 0.0484 \\
\hline & Normal & 167 & 349 & 0.479 & $48 \%$ & 0.454 & 0.42505 & 0.5323 \\
\hline
\end{tabular}

Note. $\mathrm{H}_{\mathrm{a}}$ is proportion $\neq 0.5$

Only 176 (48\%) were normal chest finding and 46 abnormal chest with consolidation (13\%). The other show variable different as shown in table (1) and figure $(5,6)$. The following observe with grounded glass opacities $(10 \%)$ and Reticular-nodular opacities (9\%). In RT-PCR positive 
patient, we found also sign nonspecific for COVID19 pneumothorax (8\%). Peripheral $36(10 \%)$ and the most common 42(12\%) Diffuse where the less frequent bilateral (3\%) then left (7\%) and right (5\%). Given the result, baseline CXR sensitivity in our experience is about $65.52 \%$.

\subsection{CXR Correlation with RT-PCR Test}

In this study, 349 patinet's records were reviewd. 168 (48.1\%) of the records were for female patients, while $181(51 \%)$ of the records were for male patients. The patient's age ranged from 14 to 98 years old. as for the symptoms, the minimum score was five, and the maximum score was 17 (see table 2).

Table 2: Descriptive Data

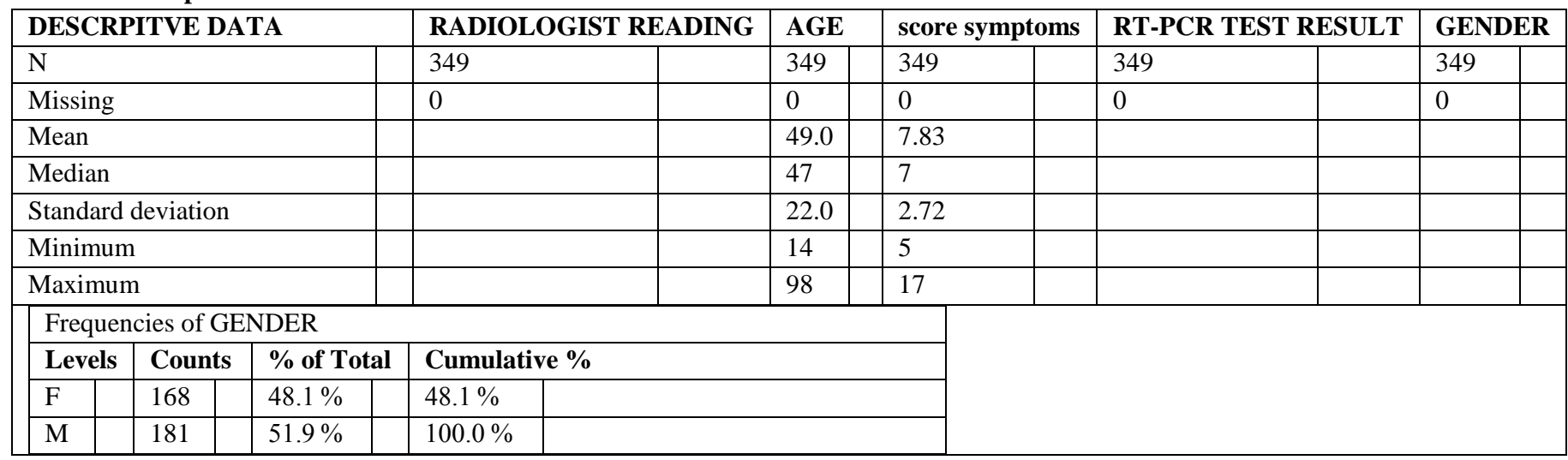

Our results showed that the account number of radiologists' negative reading (i.e., not covide19) was $47 \%$ of the sample size, and the positive reading (i.e., covid19) was $52 \%$ of the sample size (see table 3). For the RT-PCR test's standard gold scale, the positive test result was $33.2 \%$, and the negative result was $66 \%$ of the sample size (see table 4 ).

Table 3: Frequency of Radiologist Reading

\begin{tabular}{|l|l|l|l|l|l|l|}
\hline Levels & Counts & \% of Total & \multicolumn{2}{l|}{ Cumulative \% } \\
\hline NOT COVIDE19 & 167 & $47.9 \%$ & $47.9 \%$ & \\
\hline COVID19 & 182 & $52.1 \%$ & $100.0 \%$ & \\
\hline
\end{tabular}

Table 4: Frequency RT-PCR TEST

\begin{tabular}{|l|l|l|l|l|l|}
\hline Levels & Counts & \% of Total & \multicolumn{2}{l|}{ Cumulative \% } \\
\hline POSITIVE & 116 & $33.2 \%$ & $33.2 \%$ & \\
\hline NEGATIVE & 233 & $66.8 \%$ & $100.0 \%$ & \\
\hline
\end{tabular}

Interestingly, the results showed that the observers recorded 76 positive covide19 results while it was expected only 60.5 . Thus, the radiologist reading was $65 \%$ higher than the expected value. as for the negative RT-PCR test, the results showed that the radiologist reading was $54.5 \%$ higher than the expected value (see table 5).

Table 5: Expected and Observe Value

\begin{tabular}{|c|c|c|c|c|}
\hline \multicolumn{5}{|l|}{ Contingency Tables } \\
\hline \multirow[b]{2}{*}{ RT-PCR TEST RESULT } & \multirow[b]{3}{*}{ Observed } & \multicolumn{2}{|c|}{ RADIOLOGIST READING } & \multirow[b]{2}{*}{ Total } \\
\hline & & NOT COVIDE19 & COVID1 & \\
\hline POSITIVE & & 40 & 76 & 116 \\
\hline & Expected & 55.5 & 60.5 & 116 \\
\hline & $\%$ within row & $34.5 \%$ & $65.5 \%$ & $100.0 \%$ \\
\hline NEGATIVE & Observed & 127 & 106 & 233 \\
\hline & Expected & 111.5 & 121.5 & 233 \\
\hline & $\%$ within row & $54.5 \%$ & $45.5 \%$ & $100.0 \%$ \\
\hline Total & Observed & 167 & 182 & 349 \\
\hline & Expected & 167.0 & 182.0 & 349 \\
\hline & $\%$ within row & $47.9 \%$ & $52.1 \%$ & $100.0 \%$ \\
\hline
\end{tabular}

As for the relationship between the x-ray reading and the rt-pcr test result, our results showed a high correlation between the variables $\left(\chi^{2}=\right.$ 12.44 , with $\mathrm{df}=1$, and $\mathrm{p}<0.001$ ). The sensitivity of $\mathrm{x}$-ray diagnosing covid19 was $65.52 \%$, while the specificity was $54.51 \%$, and accuracy of radiologists reading was $58.17 \%$. Furthermore, the positive predictive value (ppv) was $41.76 \%$ and the negative predictive value (npv) was $76.05 \%$. finally, the false positive rate (type-i error (alpha) was $45.49 \%$, and the false negative rate (type-ii error (beta) was $34.48 \%$ (see table 6 ).

Table 6: Correlation Statistic

\begin{tabular}{|l|l|l|l|l|l|}
\hline & Gold Std: PCR & & & Xray & \\
\hline Xray & positive & negative & Total & Sensitivty & 0.655172 \\
\hline Positive & 76 & 106 & 182 & Specificity & 0.545064 \\
\hline Negative & 40 & 127 & 167 & Accuracy & 0.581662 \\
\hline
\end{tabular}




\begin{tabular}{|l|l|l|l|l|l|}
\hline Total & 116 & 233 & 349 & PPV & 0.417582 \\
\hline & & & & NPV & 0.760479 \\
\hline False Positive Rate & & Type-I error(Alpha) $=$ & 0.454936 \\
\hline False Negative Rate & \multicolumn{2}{|l|}{ Type-II error(Beta)= } & 0.344828 \\
\hline \multicolumn{2}{|l|}{ RT-PCR test and Xray are highly correlated (Pearson Chi-Square $=12.44$ with df=1 and p-value <0.001) } & & \\
\hline
\end{tabular}

Our study aimed to investigate the association between CXRP and RT-PCR test and if the CXRP is a reliable tool to diagnose COVID19 when the RT-PCR test is unavailable. The main finding can be summarized as follow: The 5-point scale for chest scorning assessment gives accurate guidance on the seriousness of inflammation. The analysis showed a high correlation between the CXRP imaging reading of radiologists and the RT-PCR test result $\left(\chi^{2}=12.44\right.$, df $=1$, and $\left.\mathrm{p}<0.001\right)$. The Sensitivity of $X$-ray in diagnosing Covid19 was $65.52 \%$, while the Specificity was $54.51 \%$, and the accuracy of the radiologist's reading was 58\%. This study's finding is aligned with seven previous studies to evaluate the sensitivity of CXR in COVID19 patients (See table 7).

Table 7: Comparison among Studies on CXR Sensitivity in COVID19 Patient

\begin{tabular}{|l|l|l|l|}
\hline Study title & Research author & No. of patient & CXR sensitivity \% \\
\hline $\begin{array}{l}\text { Chest X-ray in new Coronavirus Disease 2019 (COVID-19) infection: } \\
\text { findings and correlation with clinical outcome }\end{array}$ & Cozzi et al (2020) & 482 & $68.1 \%$ \\
\hline $\begin{array}{l}\text { Determinants of Chest X-Ray Sensitivity for COVID- 19: A Multi- } \\
\text { Institutional Study in the United States }\end{array}$ & Stephanie, MD (2020) & 508 & $55.0 \%$ \\
\hline $\begin{array}{l}\text { Frequency and Distribution of Chest Radiographic Findings in Patients } \\
\text { Positive for COVID-19 }\end{array}$ & Wong et al (2020) & 64 & $68.8 \%$ \\
\hline $\begin{array}{l}\text { Diagnostic impact of bedside chest X-ray features of 2019 novel } \\
\text { coronavirus in the routine admission at the emergency department: case } \\
\text { series from Lombardy region }\end{array}$ & Ippolito et al (2020) & 204 & $57.0 \%$ \\
\hline $\begin{array}{l}\text { Diagnostic Performance of Chest X-Ray for COVID-19 Pneumonia } \\
\text { During the SARS-CoV-2 Pandemic in Lombardy, Italy }\end{array}$ & Schiaffino et al (2020) & 408 & $89.0 \%$ \\
\hline Clinical Characteristics of Coronavirus Disease 2019 in China & Guan et al (2020) & 636 & $41.7 \%$ \\
\hline $\begin{array}{l}\text { Baseline chest X-ray in coronavirus disease 19 (COVID-19) patients: } \\
\text { association with clinical and laboratory data }\end{array}$ & Gatti et al (2020) & 260 & $61.2 \%$ \\
\hline $\begin{array}{l}\text { Is the portable chest radiographic more reliable to reveal covid19 in } \\
\text { highly suspicion patient before real-time reverse transcription polymerase } \\
\text { chain reaction (RT-PCR) test }\end{array}$ & Our study (2020) & 349 & $65.52 \%$ \\
\hline
\end{tabular}

Radiographic Measurement of Lung Edema (RALE) was used by Cozzi and his colleagues (2020) similar to radiographic score system that been used on our study. In their study, the CXR was assigned a score between 0 and 48, ranging from the absence of any pathological indication (score 0) to lung parenchyma's full pathological presence (score 48). The radiological features of COVID-19 patients with patchy or diffuse reticular-nodular opacities and convergence (Cozzi et al., 2020). The only difference is that the RT-PCR test was executed 2 and 15 days after the onset of symptoms, with more advanced lung symptoms in people around the 10th day of illness and only include on his sample patient with positive result of RT-PCR test. Still, in our study, the time of the RT-PCR test was executed within the 24-hour maximum which make our result lower because most of the RTPCRT test result at first time might be negative related to the onset system on the early days of illness.

Our study results reinforced Wong and his colleagues (2020), in which their study showed observed baseline RT-PCR detection rate, using RT-PCR findings as to the Gold standard, 58/64, 91\% sensitivity [95\% CI: 83-97\%]) above baseline CXR (44/64, 69\% sensitivity (95\% CI: 56-80\%) ( $\mathrm{p}=0.009)$ (Wong et al., 2020). The mean time between positive RT-PCR and negative RTPCR has been $8 \pm 7$ days ( $n=23$, range 1-24 days). Initially, their study's result brought into line with our in the mean period was $6 \pm 5$ days between positive CXR and negative CXR, and the most common result was consolidation accompanied by Multilobar ground glass. The distribution was a peripheral and lower zone, with a $50 \%$ bilateral participation. There was no typical pleural outbreak; thus, the CXR outcomes were severe 10-12 days from the outcome's date even though on our study the onset symptoms count when the patient admitted at ER the result remain sold because we are using RT-PCRT as gold stander for COVID19 confirmation.

Amusingly, this study's results are also aligned with Schiaffino et al. (2020) on the CXR initial results. In their results they reported a sensitivity of $89.0 \%$ (Schiaffino et al., 2020). With RT-PCR, the adoption of CXR to triage patients with suspected SARS-CoV-2 infection facilitates a stable and effective workflow, counteracting possible false-negative RT-PCR. Our results found the CXR was a useful tool to manage the patient on triage and give them better treatment.

Gatti et al. (2020) observed that CXR has low sensitivity comparing the negative COVID19 group with positive COVID19 group, and that was aligned with our study on the important time between the onset symptom and execution of CXR on highly suspicion COVID19 patient specially those who highly alert with high score symptoms comes with hypertension and dyspnea were common found on positive COVID19 (Gatti et al., 2020). The time between the execution of CXR and the onset of symptoms is a significant indicator of positive CXR. Using RT-PCR as the gold standard, the CXR sensitivity was $61.1 \%$ (95\% CI 55-67\%), tremendously close to our result of CXR sensitivity. Airspace opacities were reported and most widely located in the peripheral and lower region, and most patients had bilateral involvement. Pleural effusion is the most common observation. The only different between our study and their study that we do not have control group negative COVID19 because all suspicious patient we deal with them as positive patient until the RT-PCR test show negative result. 
Fascinating, the same result showed by Stephine and his colleagues (2020). Their results indicated that the magnitude and sensitivity of positive COVID-19 CXR findings improve over time, with a marked rise in sensitivity following the onset of symptoms on Day 6. The time from disease onset to dyspnea, sepsis, and acute respiratory distress syndrome in positive COVID-19 patients has been recorded as seven days, nine days, and 12 days respectively, coinciding with the known time course pathophysiological decline in patients (Stephanie et al., 2020). Unlike Stephanie and his colleague (2020), in our study, the time of onset symptom is recorded on the first day of patient admission to the ER and before the admission day of the medical record remains unknown although the same techniques were similar in our study our result showed higher number than (Stephanie et al., 2020). The reason of having higher number in our study could be referred to most patient with suspicious COVID 19 did not have serial CXR approaches with CT for confirmed pathology and our gold stander was RT-PCR test to confirm the positive COVID 19 thus make different on the result and exclude any doubt of respiratory disease similar signs to COVID19.

A study conducted by Ippolito and his colleagues (2020) found that the overall chest X-ray exposure for SARS-CoV-2 pneumonia was $57 \%$. According to them, the sensitivity was greater when symptoms occurred more than five days ago, at the cost of reduced specificity, and significantly higher in older patients than younger patients (Ippolito et al., 2020). The patients with symptoms lasting more than five days showed a higher number of reticular and alveolar opacities than patients with symptoms onset less than five days Moreover all elderly patient score high sensitivity because most of them has medical history of lung disease. Ippolito and his colleague (2020) contradict with our result because they focus on the prognosis of the disease with approved positive COVID19 patient who admitted in ER as inpatient not suspicious.

Guan and his colleagues (2020) found that the clinical characteristics of Covid-19 mimic those of SARS-CoV. Fever and cough were the dominant symptoms, and gastrointestinal symptoms were uncommon. The absence of fever in Covid-19 is more frequent. The degree of severity of Covid-19 on the first admission is classified as non sensitive and severe patients. They reported that the patients with a severe disorder were older than patients with non-severe disability by a total of 7 years. Moreover, in patients with severe disease, the existence of some coexisting conditions was more frequent than in patients with non-severe disease (Guan et al., 2020). Our study, reinforced their study because we found $30 \%$ differently most of the patients having high score symptoms from 8 and above with medical history of lung disease along with fever, headache, running nose, diarrhea, active chest pain, shortness of breathing, and vomiting especially on an elderly patient.

Finally, our research has several limitations that could affect the results. First of all, having retrospective data and the absence of a non-COVID-19 control group in the prognostic score study decreases the sensitivity and reliability of the CXR. The second limitation is related to the radiologist's experience. Moreover, our radiologists in this research have more than ten years of experience and display a confounding predictor if the radiologist has low expertise. Finally, the lack of correlation between the RALE score and the patients' chronic conditions is often a drawback. Yet, specific health findings were only available in a small range of subjects and the accurate information on medical records about the patient onset symptoms

\section{Conclusions}

Our research's main goal was to investigate the reliability of CXR portable in diagnosing COVID19 before the RT-PCR test. Moreover, we were investigating the correlation between CXRP and RT-PCRT test. The RT-PCR test was used as the golden standard in this study to correlate with the CXRP image reporting result. Using the score system radiologist reading allowed us to show the most familiar future of COVD19.

Our research findings showed that the CXRP imaging could detect COVID-19 infection in symptomatic patients and could be a valuable addition to RT-PCR testing. In an inpatient ED environment where availability of test kits, laboratory equipment, and laboratory personnel was compromised and risks delaying patient treatment and workflow, serial CXRP could theoretically be used as an adjunct diagnostic function and monitoring in patients suspected of having COVID-19.

\section{Ethics approval and consent to participate}

Ethical approval obtained from the institutional board Prince Sultan Medical Military Hospital City to wave the consent forms since it is a retrospective study. Only the investigators will have data access to the patients medical files.

\section{List of abbreviations}

$\begin{array}{ll}\text { CXR } & \text { Chest x-ray } \\ \text { CXRP } & \text { Chest x-ray portable } \\ \text { ACR } & \text { American College Radiology } \\ \text { PR-PCT } & \text { reverse transcription-polymerase chain reaction } \\ \text { MOH } & \text { Ministry of Saudi health } \\ \text { CT } & \text { Computed tomography } \\ \text { SARS-CoV-2 } & \text { Severe acute respiratory syndrome coronavirus 2 } \\ \text { IRB } & \text { Institutional board approval } \\ \text { ED } & \text { Emergency department } \\ \text { ICU } & \text { Intensive care unite } \\ \text { PACS } & \text { Picture archiving communication system } \\ \text { PA } & \text { Posterior anterior } \\ \text { AP } & \text { Anterior posterior } \\ \text { PSMMC } & \text { Prince Sultan Military Medical City }\end{array}$

\section{Conflicts of Interest}

"The author(s) declare(s) that there is no conflict of interest regarding the publication of this paper."

\section{Funding Statement}

No funding

\section{Authors' contributions}

BB main author literature search,study design, research proposal for IRB. Data abstraction and collection, data analysis and Writing up the final research thesis follow the rule of PSMMC.

NB proof reading and data analysis

NQ image interpretation

ANG image interpretation

RM Review literature search and final research thesis 


\section{References}

[1] Akçay, Ş., Özlü, T., \& Yılmaz, A. (2020). Radiological approaches to COVID-19 pneumonia. Turk J Med Sci, 50(Si-1), 604-610. doi:10.3906/sag-2004-160

[2] Bharadwaz, A., \& Langfeldt, S. (2020). Morphological patterns and distributions in portable chest radiographs of COVID-19-positive cases admitted to a tertiary care hospital: An early experience from Scandinavia. J Med Imaging Radiat Oncol. doi:10.1111/1754-9485.13123

[3] Borghesi, A., \& Maroldi, R. (2020). COVID-19 outbreak in Italy: experimental chest X-ray scoring system for quantifying and monitoring disease progression. Radiol Med, 125(5), 509-513. doi:10.1007/s11547-020-01200-3

[4] Carotti, M., Salaffi, F., Sarzi-Puttini, P., Agostini, A., Borgheresi, A., Minorati, D.,... Giovagnoni, A. (2020). Chest CT features of coronavirus disease 2019 (COVID19) pneumonia: key points for radiologists. Radiol Med, 125(7), 636-646. doi:10.1007/s11547-020-01237-4

[5] Cozzi, D., Albanesi, M., Cavigli, E., Moroni, C., Bindi, A., Luvarà, S.,... Miele, V. (2020). Chest X-ray in new Coronavirus Disease 2019 (COVID-19) infection: findings and correlation with clinical outcome. Radiol Med, 125(8), 730-737. doi:10.1007/s11547-020-01232-9

[6] de Barry, O., Obadia, I., El Hajjam, M., \& Carlier, R. Y. (2020). Chest-X-ray is a mainstay for follow-up in critically ill patients with covid-19 induced pneumonia. $\begin{array}{llll}\text { Eur J } & \text { Radiol, } & 109075 .\end{array}$ doi:10.1016/j.ejrad.2020.109075

[7] Durrani, M., Haq, I. U., Kalsoom, U., \& Yousaf, A. (2020). Chest X-rays findings in COVID 19 patients at a University Teaching Hospital - A descriptive study. Pakistan journal of medical sciences, 36(COVID19-S4), S22-S26. doi:10.12669/pjms.36.COVID19-S4.2778

[8] Fatima, S., Ratnani, I., Husain, M., \& Surani, S. (2020). Radiological Findings in Patients with COVID-19. Cureus, 12(4).

[9] Gatti, M., Calandri, M., Barba, M., Biondo, A., Geninatti, C., Gentile, S.,... Faletti, R. (2020). Baseline chest X-ray in coronavirus disease 19 (COVID-19) patients: association with clinical and laboratory data. Radiol Med, 125(12), 1271-1279. doi:10.1007/s11547020-01272-1

[10] Guan, W. J., Ni, Z. Y., Hu, Y., Liang, W. H., Ou, C. Q., He, J. X.,... Zhong, N. S. (2020). Clinical Characteristics of Coronavirus Disease 2019 in China. N Engl J Med, 382(18), 1708-1720. doi:10.1056/NEJMoa2002032

[11] HEALTH, M. O. S. (2020). The COVID-19 guidelines were done with the best available data and evidence. Retrieved from https://www.moh.gov.sa/Ministry/MediaCenter/Publicati ons/Documents/Coronavirus-Disease-2019-Guidelinesv1.2.pdf

[12] Hu, L., \& Wang, C. (2020). Radiological role in the detection, diagnosis and monitoring for the coronavirus disease 2019 (COVID-19). Eur Rev Med Pharmacol Sci, 24(8), 4523-4528. doi:10.26355/eurrev_202004_21035

[13] Ippolito, D., Pecorelli, A., Maino, C., Capodaglio, C., Mariani, I., Giandola, T.,... Sironi, S. (2020). Diagnostic impact of bedside chest X-ray features of 2019 novel coronavirus in the routine admission at the emergency department: case series from Lombardy region. Eur J Radiol, 129, 109092. doi:10.1016/j.ejrad.2020.109092
[14] Jacobi, A., Chung, M., Bernheim, A., \& Eber, C. (2020). Portable chest X-ray in coronavirus disease-19 (COVID19): A pictorial review. Clin Imaging, 64, 35-42. doi:10.1016/j.clinimag.2020.04.001

[15] Khan, T., Lopez, T., Khan, T., Ali, A., Syed, S., Patil, P., \& Hatoum, A. (2020). Re: a British Society of Thoracic Imaging statement: considerations in designing local imaging diagnostic algorithms for the COVID-19 pandemic. Clin Radiol, 75(8), 636. doi:10.1016/j.crad.2020.05.009

[16] Kooraki, S., Hosseiny, M., Myers, L., \& Gholamrezanezhad, A. (2020). Coronavirus (COVID-19) Outbreak: What the Department of Radiology Should Know. J Am Coll Radiol, 17(4), 447-451. doi:10.1016/j.jacr.2020.02.008

[17] Liu, K., Fang, Y. Y., Deng, Y., Liu, W., Wang, M. F., Ma, J. P.,... Liu, H. G. (2020). Clinical characteristics of novel coronavirus cases in tertiary hospitals in Hubei Province. Chin Med J (Engl), 133(9), 1025-1031. doi:10.1097/cm9.0000000000000744

[18] Lovas, A., Hankovszky, P., Korsós, A., Kupcsulik, S., Molnár, T., Szabó, Z., \& Babik, B. (2020). [Importance of the imaging techniques in the management of COVID19-infected patients]. Orv Hetil, 161(17), 672-677. doi:10.1556/650.2020.31814

[19] Pereira, R. M., Bertolini, D., Teixeira, L. O., Silla, C. N., Jr., \& Costa, Y. M. G. (2020). COVID-19 identification in chest X-ray images on flat and hierarchical classification scenarios. Comput Methods Programs Biomed, 194, 105532. doi:10.1016/j.cmpb.2020.105532

[20] RADIOLOGY, A. A. C. O. (2020). Recommendations for the use of Chest Radiography and Computed Tomography (CT) for Suspected COVID-19 Infection. Retrieved from https://www.acr.org/Advocacy-andEconomics/ACR-Position-

Statements/Recommendations-for-Chest-Radiographyand-CT-for-Suspected-COVID19-Infection

[21] Schiaffino, S., Tritella, S., Cozzi, A., Carriero, S., Blandi, L., Ferraris, L., \& Sardanelli, F. (2020). Diagnostic Performance of Chest X-Ray for COVID-19 Pneumonia During the SARS-CoV-2 Pandemic in Lombardy, Italy. J Thorac Imaging. doi:10.1097/rti.0000000000000533

[22] Singer, A. J., Morley, E. J., Meyers, K., Fernandes, R., Rowe, A. L., Viccellio, P.,... Henry, M. C. (2020). Cohort of 4404 Persons Under Investigation for COVID19 in a NY Hospital and Predictors of ICU Care and Ventilation. Annals of Emergency Medicine.

[23] Stephanie, S., Shum, T., Cleveland, H., Challa, S. R., Herring, A., Jacobson, F. L.,... Hammer, M. M. (2020). Determinants of Chest X-Ray Sensitivity for COVID19: A Multi-Institutional Study in the United States. Radiology: Cardiothoracic Imaging, 2(5), e200337. doi:10.1148/ryct.2020200337

[24] Toussie, D., Voutsinas, N., Finkelstein, M., Cedillo, M. A., Manna, S., Maron, S. Z., Gupta, Y. S. (2020). Clinical and Chest Radiography Features Determine Patient Outcomes In Young and Middle Age Adults with COVID-19. Radiology, 0(0), 201754. doi:10.1148/radiol.2020201754

[25] Vancheri, S. G., Savietto, G., Ballati, F., Maggi, A., Canino, C., Bortolotto, C., Preda, L. (2020). Radiographic findings in 240 patients with COVID-19 pneumonia: time-dependence after the onset of 
symptoms. European Radiology. doi:10.1007/s00330020-06967-7

[26] Wong, A., Lin, Z. Q., Wang, L., Chung, A. G., Shen, B., Abbasi, A.,... Duong, T. Q. (2020). Towards computeraided severity assessment: training and validation of deep neural networks for geographic extent and opacity extent scoring of chest X-rays for SARS-CoV-2 lung disease severity. arXiv preprint arXiv:2005.12855.

[27] Yanagawa, Y., Ohsaka, H., Oode, Y., \& Omori, K. (2019). A case of fatal trauma evaluated using a portable X-ray system at the scene. J Rural Med, 14(2), 249-252. doi: $10.2185 / \mathrm{jrm} .3002$

[28] Ye, Z., Zhang, Y., Wang, Y., Huang, Z., \& Song, B. (2020). Chest CT manifestations of new coronavirus disease 2019 (COVID-19): a pictorial review. Eur Radiol, 30(8), 4381-4389. doi:10.1007/s00330-02006801-0

[29] Yin, Z., Kang, Z., Yang, D., Ding, S., Luo, H., \& Xiao, E. (2020). A Comparison of Clinical and Chest CT Findings in Patients With Influenza A (H1N1) Virus Infection and Coronavirus Disease (COVID-19). AJR Am J Roentgenol, 215(5), 1065-1071. doi:10.2214/ajr.20.23214

[30] Yu, Y., \& Chen, P. (2020). Coronavirus Disease 2019 (COVID-19) in Neonates and Children From China: A Review. Front Pediatr, 8, 287. doi:10.3389/fped.2020.00287

[31] Wong, A., Lin, Z. Q., Wang, L., Chung, A. G., Shen, B., Abbasi, A.,... Duong, T. Q. (2020). Towards computeraided severity assessment: training and validation of deep neural networks for geographic extent and opacity extent scoring of chest X-rays for SARS-CoV-2 lung disease severity. arXiv preprint arXiv:2005.12855

[32] Tietze, F., Vimalnath, P., Aristodemou, L., \& Molloy, J. (2020). Crisis-Critical Intellectual Property: Findings from the COVID-19 Pandemic. Available at SSRN 3569282 .

[33] Sinha, I. (2020). Guidance for the clinical management of children admitted to hospital with suspected COVID19. British Paediatric Respiratory Society.

[34] Sohail, S. (2020). Rational and practical use of imaging in COVID-19 pneumonia. Pakistan journal of medical sciences, 36(Covid19-s4), S130-s133. doi:10.12669/pjms.36.COVID19-S4.2760

[35] Shi, H., Han, X., Jiang, N., Cao, Y., Alwalid, O., Gu, J.,... Zheng, C. (2020). Radiological findings from 81 patients with COVID-19 pneumonia in Wuhan, China: a descriptive study. Lancet Infect Dis, 20(4), 425-434. doi:10.1016/s1473-3099(20)30086-4

[36] Sayiner, A., Cinkooglu, A., Tasbakan, M. S., Basoglu Ö, K., Ceylan, N., Savas, R.,... Özhan, M. H. (2020). Radiographic examination of the chest and COVID-19. Ann R Coll Surg Engl, 102(5), 334. doi:10.1308/rcsann.2020.0099

[37] Rousan, L. A., Elobeid, E., Karrar, M., \& Khader, Y. (2020). Chest X-ray findings and temporal lung changes in patients with COVID-19 pneumonia. BMC Pulm Med, 20(1), 245. doi:10.1186/s12890-020-01286-5

[38] Rorat, M., Zińczuk, A., Szymański, W., Simon, K., \& Guziński, M. (2020). Usefulness of a portable chest radiograph in the initial diagnosis of coronavirus disease 2019. Pol Arch Intern Med, 130(10), 906-909. doi:10.20452/pamw.15512

[39] Lovas, A., Hankovszky, P., Korsós, A., Kupcsulik, S., Molnár, T., Szabó, Z., \& Babik, B. (2020). [Importance of the imaging techniques in the management of COVID19-infected patients]. Orv Hetil, 161(17), 672-677. doi:10.1556/650.2020.31814

[40] Liu, K., Fang, Y. Y., Deng, Y., Liu, W., Wang, M. F., Ma, J. P.,... Liu, H. G. (2020). Clinical characteristics of novel coronavirus cases in tertiary hospitals in Hubei Province. Chin Med J (Engl), 133(9), 1025-1031. doi:10.1097/cm9.0000000000000744

[41] Kundu, S., Elhalawani, H., Gichoya, J. W., \& Charles E. Kahn, J. (2020). How Might AI and Chest Imaging Help Unravel COVID-19's Mysteries? Radiology: Artificial Intelligence, 2(3), e200053. doi:10.1148/ryai.2020200053

[42] Kooraki, S., Hosseiny, M., Myers, L., \& Gholamrezanezhad, A. (2020). Coronavirus (COVID-19) Outbreak: What the Department of Radiology Should Know. J Am Coll Radiol, 17(4), 447-451. doi:10.1016/j.jacr.2020.02.008

[43] Fu, L., Wang, B., Yuan, T., Chen, X., Ao, Y., Fitzpatrick, T.,... Zou, H. (2020). Clinical characteristics of coronavirus disease 2019 (COVID-19) in China: A systematic review and meta-analysis. J Infect, 80(6), 656665. doi:10.1016/j.jinf.2020.03.041

[44] Hu, L., \& Wang, C. (2020). Radiological role in the detection, diagnosis and monitoring for the coronavirus disease 2019 (COVID-19). Eur Rev Med Pharmacol Sci, 24(8), 4523-4528. doi:10.26355/eurrev_202004_21035

[45] Fatima, S., Ratnani, I., Husain, M., \& Surani, S. (2020). Radiological Findings in Patients with COVID-19. Cureus, 12(4), e7651. doi:10.7759/cureus.7651 